\title{
Infarto do miocárdio sem doença coronariana obstrutiva (Síndrome MINOCA)
}

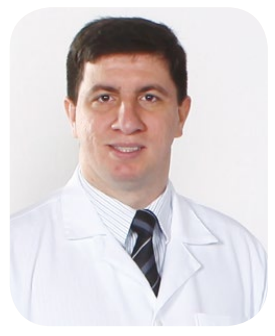

\author{
Gilson Feitosa-Filho¹, Julio Braga², Joberto Sena', Gilson Feitosa'
}

Desde o surgimento da cineangiocoronariografia ${ }^{1} \mathrm{e}$ sua utilização na fase aguda do infarto ${ }^{2} e$, principalmente, depois do surgimento de marcadores sensíveis séricos de morte de cardiomiócito, como as troponinas, registram-se casos que possuem características clínicas de apresentação de infarto sem a presença de obstruções significativas das coronárias, isto é, lesões $<50 \%$. Admitindo várias etiologias e mecanismos, passaram a ser mais recentemente cunhadas em literatura internacional como síndrome MINOCA (Myocardial Infaction with Nonobstructive Coronary Arteries) ${ }^{3}$. Estes casos representam cerca de 5 a $10 \%$ dos casos de infarto do miocárdio e mais frequentemente são vistos em pacientes mais jovens e com ligeiro predomínio do sexo feminino. Vide figura 1.

\begin{tabular}{|c|c|c|c|}
\hline & \multicolumn{2}{|c|}{ No./Total (\%) } & \multirow[b]{2}{*}{$P$ Value } \\
\hline & Women & Men & \\
\hline \multicolumn{4}{|l|}{ Acute coronary syndrome } \\
\hline GUSTO2 & $343 / 1768(19.4)$ & $394 / 4638(8.4)$ & $<.001$ \\
\hline $\mathrm{TIMI} 18^{3}$ & $95 / 555(17)$ & $99 / 1091$ (9) & $<.001$ \\
\hline Unstable angina ${ }^{2}$ & $252 / 826(30.5)$ & $220 / 1580(13.9)$ & $<.001$ \\
\hline TIMI IIIa ${ }^{6}$ & $30 / 113(26.5)$ & $27 / 278(8.3)$ & $<.001$ \\
\hline Ml without ST-segment elevation² & $41 / 450(9.1)$ & $55 / 1299(4.2)$ & .001 \\
\hline Ml with ST-segment elevation ${ }^{2}$ & $50 / 492(10.2)$ & $119 / 1759(6.8)$ & .02 \\
\hline
\end{tabular}

Figura 1. Prevalência de artérias coronárias sem obstruções significativas em distintos cenários de síndrome coronariana aguda em ambos os gêneros. Adaptado de Bugiardini R , Bairey Merz CN. JAMA 2005;293(4):477-84

Geralmente encerram um prognóstico melhor ${ }^{4,5}$ do que os casos de IAM com obstrução coronariana, embora que uma taxa de mortalidade de 4,7\%, descrita em um ano, não seja desprezível. ${ }^{6}$

Condição básica para o seu diagnóstico é a suspeita de infarto do miocárdio, elevação de troponina com padrão típico de curva de ascensão de queda e uma cinecoronariografia que demonstre a ausência de obstruções no interior das coronárias ou, em havendo alguma, que seja menor que $50 \%$ da luz do vaso.

Condições assim podem estar relacionadas a distintas causas, como pode ser apreciado no Quadro 1. 


\begin{tabular}{l}
\hline Quadro 1. Causas de infarto do miocárdio sem obstrução das coronárias (MINOCA) \\
\hline CARDÍACAS \\
\hline Coronárias: \\
\hline rotura de placa de ateroma \\
\hline trombose espontânea da coronária ou embolismo \\
\hline disfunção microvascular \\
\hline dissecção de coronária \\
\hline espasmo coronariano \\
\hline Doenças do miocárdio \\
\hline miocardite \\
\hline cardiomiopatia de takotsubo \\
\hline NÃO-CARDÍACAS \\
\hline embolismo pulmonar \\
\hline insuficiência renal \\
\hline
\end{tabular}

O elemento central para o diagnóstico é o de uma elevação, em curva de ascenção e queda da troponina, além de coronariografia sem doença obstrutiva significativa, num contexto em que infarto deva ser considerado pela presença de: dor sugestiva de isquemia miocárdica; ou alteração eletrocardiográfica, com surgimento de ondas $Q$, novo bloqueio de ramo ou alteração da repolarização miocárdica; ou demonstração de alteração segmentar da parede miocárdica em método de imagem.

Tais alterações poderão ocorrer em consequência de um embolismo pulmonar agudo ou no curso de insuficiência renal, em cujos casos o reconhecimento das condições básicas existentes levam a medidas de cuidados voltados para essas.

Também entre as condições cardíacas consideradas ao tempo da realização da coronariografia, em se tratando da miocardiopatia catecolaminérgica (takotsubo), o padrão já visto por método de imagem, geralmente o ecocardiograma com envolvimento simultâneo de várias paredes, ou mesmo com a ventriculografia realizada durante o cateterismo cardíaco, conduzem ao diagnóstico da situação com subsequente estratégia de tratamento.

Ocasionalmente, a idade mais jovem do acometido, na ausência de fatores de risco para a doença coronariana aterosclerótica, especialmente se associada a dados nosológicos de infecção viral recente, febre ou dor pericárdica (ou pleurítica) associada, leva ao diagnóstico presumível de miopericardite com as condutas subsequentes diagnósticas e terapêuticas atinentes.

Quando este não é o caso, isto é, quando a causa aparente de um infarto sem obstrução coronária ainda não está fortemente sugerida ao tempo da coronario- grafia, a obtenção em seguida de uma ressonância magnética cardíaca apontará para possível miocardite em cerca de $30 \%$ dos casos. ${ }^{7}$

Há quem prefira chamar MINOCA aos casos em que sua origem se deva à alteração das coronárias, reservando o termo TP-NOCA (Troponin Positive in Non-Obstructive Coronary Artery) para os demais.

Considerando-se a origem primariamente coronária do infarto ${ }^{8}$ em doença coronariana não obstrutiva, muitos mecanismo são possíveis.

Uma cuidadosa revisão da coronariografia se impõe diante da possibilidade, às vezes sutil, da existência de uma dissecção, ou de um de pequeno trombo.

A cinecoronariografia, nestes casos de obstrução < $50 \%$, revela dois padrões de achados: em metade dos casos há obstruções entre 30 e 50\%. E nos demais, chamados de sem aterosclerose, que representam a outra metade de casos, a luminografia revela lesões mínimas, abaixo de $30 \%$, ou ausência de qualquer lesão evidenciável pela cinecoronariografia.

Estudos com ultrassonografia intracoronária (USIC) revelam naqueles que apresentam aterosclerose - entre 30 e $50 \%$ - evidência de rotura de placa em $70 \%$ dos casos, estabelecendo, desse modo, uma convincente demonstração do papel aterotrombótico, mesmo sem obstruções significativas, como sempre se suspeitou. ${ }^{9}$

A pesquisa de mecanismos de espasmos, quando realizada, deverá ser após recuperação do infarto. Observa-se uma prática pouco frequente de sua utilização, exceto em grupos com interesse de pesquisa no assunto.

$\mathrm{Na}$ fase aguda do processo, embora não conste de diretrizes estabelecidas sobre o tema, geralmente 
como ilação do que ocorre no IAM habitual com obstrução coronariana, os mesmos recursos medicamentosos são utilizados ${ }^{10}$, sendo que o tratamento difere apenas pela não utilização de estratégias de revascularização.

Já a longo prazo, a melhor evidência existente advém de um estudo recentemente publicado e realizado no contexto do registro Sueco de Registro SWEDEHEART (the Swedish Web-system for Enhancement and Development of Evidence-based care in Heart disease
Evaluated According to Recommended Therapy), onde dos 199.162 casos de IAM, de junho 2003 até junho 2013, e acompanhados até dezembro 2013, 9.466 casos apresentavam coronariografia sem lesões obstrutivas significativas.

Neste estudo observacional houve a seleção de um grupo comparativo dentro do conceito de escore de propensão e os resultados podem ser apreciados na figura 2.
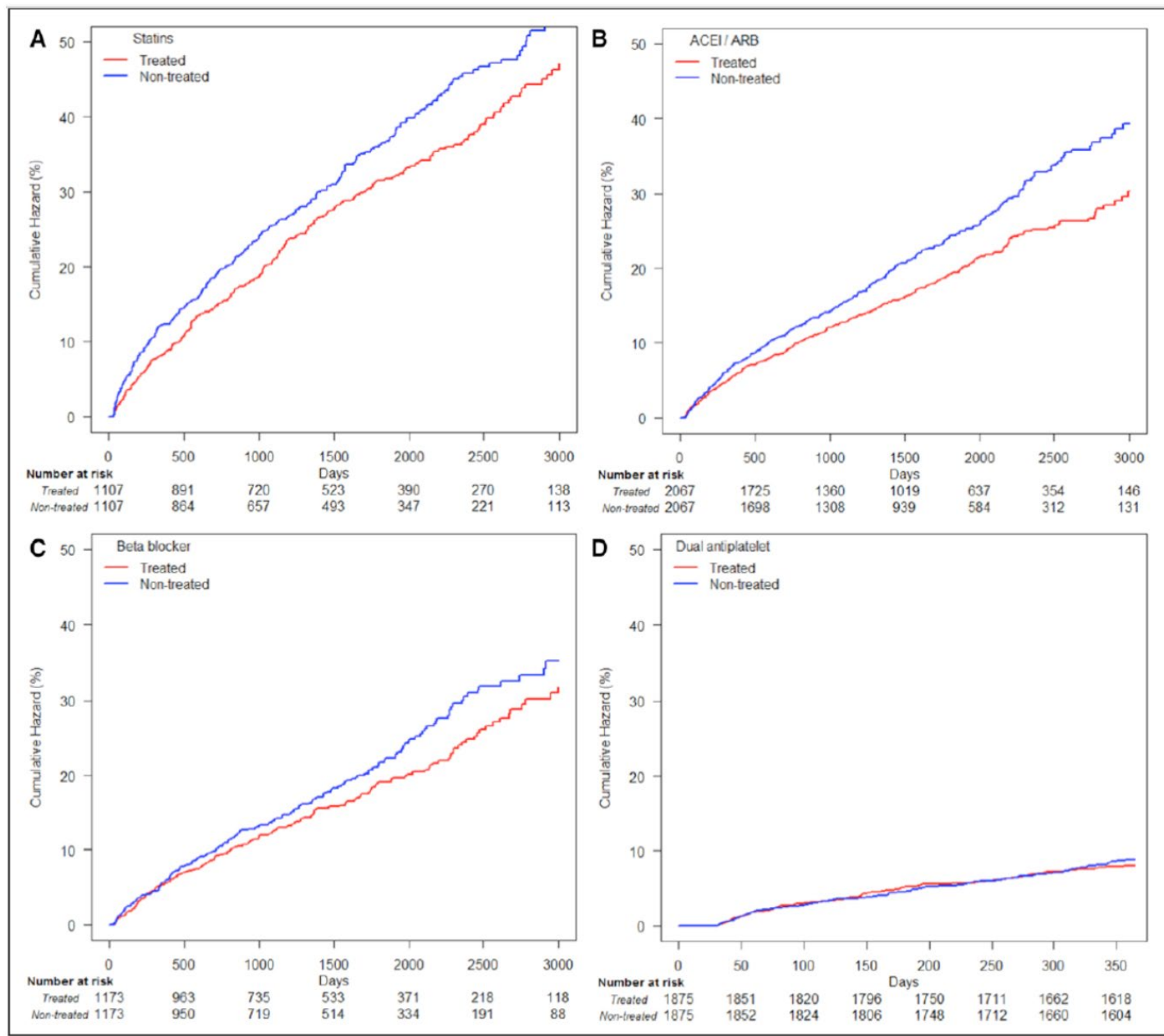

\begin{tabular}{|l|c|c|c|c|}
\hline & \multicolumn{1}{|c|}{ Statins } & ACEI/ARB & $\boldsymbol{\beta}$-Blockers & DAPT* \\
\hline Women & $0.80(0.69-0.94) n=4550$ & $0.75(0.64-0.88) n=3557$ & $0.89(0.73-1.08) n=3817$ & $0.86(0.68-1.09) n=4995$ \\
\hline Men & $0.70(0.57-0.85) n=2967$ & $0.95(0.78-1.16) n=2347$ & $0.82(0.64-1.06) n=2545$ & $0.92(0.68-1.25) n=3123$ \\
\hline$<70 y$ & $0.73(0.60-0.90) n=4718$ & $0.96(0.80-1.15)+n=3861$ & $0.88(0.70-1.10) n=4192$ & $0.83(0.60-1.13) n=5066$ \\
\hline$\geq 70 y$ & $0.85(0.72-0.99) n=2799$ & $0.74(0.63-0.87)+n=2043$ & $0.83(0.68-1.03) n=2170$ & $0.96(0.76-1.21) n=3052$ \\
\hline
\end{tabular}

Figura 2. Efeito do tratamento em desfechos cardiovasculares maiores (MACE), com estatina, IECA/BRA, betabloqueadores ou dupla terapia antiplaquetária, comparando-se em população com MINOCA os que fizeram uso do agente farmacológico com os que não fizeram, pareados num modelo de escore de propensão. 
Observa-se que de forma mais consistente, em pacientes com MINOCA, os efeitos mais significativos são obtidos com o emprego a longo prazo das estatinas que se mostraram relevantes em todos os subgrupos avaliados. Houve uma tendência de benefícios com os IECA/BRA e betabloqueadores. Demonstrou-se também que terapia antiplaquetária dupla não foi benéfica nesta população.

Há que se considerar, no entanto, que a síndrome MINOCA envolve um conjunto muito heterogêneo de possíveis etiologias do infarto com coronárias sem obstruções e que, para algumas etiologias, espera-se uma ação importantíssima da dupla antiagregação plaquetaria. Espera-se, por exemplo, que a dupla antiagregação plaquetária seja muito mais importante na dissecção espontânea de coronárias que na Síndrome de Takotsubo, por exemplo. No presente estudo, não foi possível dividir os pacientes por essas ou outras prováveis etiologias.

Tal conhecimento é muito relevante tanto para os pacientes como para os médicos que os tratam, pois há documentada tendência a ser menos complacente com o emprego de fármacos comprovadamente benéficos nesse grupo de pacientes portadores de coronárias sem doença obstrutiva, mesmo que tenha sido acometida por infarto.

\section{CONCLUSÃO}

Diante de quadros de MINOCA, depois de uma coronariografia: verificar minuciosamente os dados angiográficos à procura de placas possivelmente rotas, ou presença de trombos ou dissecções. Excluir miocardite e Takotsubo, principalmente com o emprego de ecocardiografia e ressonância magnética, e considerar a pesquisa de trombofilia. Usar terapêutica de AAS, IECA/BRA, estatina. A depender de cada caso, pode-se considerar o uso do $2^{\circ}$ antiagregante plaquetário. $\mathrm{Na}$ recorrência de angina, considerar o uso empírico de antagonista do cálcio.

Raramente, testes provocativos de isquemia são utilizados.

\section{REFERÊNCIAS}

1. Sones FM Jr. Cine-coronary arteriography. Ohio State Med J. 1962;58:1018-9.

2. Rentrop KP, Blanke H, Karsch KR, Kreuzer H. Initial Experience with Transluminal Recanalization of the Recently Occluded Infarct-Related Coronary Artery in Acute Myocardial Infarction - Comparison with Conventionally Treated Patients. Clin Cardiol, 1979; 2:92-105.

3. Agewall S, Beltrame JF, Reynolds HR, Niessner A, Rosano G, Caforio AL, De Caterina R, Zimarino
M, Roffi M, Kjeldsen K, Atar D, Kaski JC, Sechtem $U$, Tornvall $P$ and Pharmacotherapy WGoC. ESC working group position paper on myocardial infarction with non-obstructive coronary arteries. Eur Heart J. 2017;38:143-153.

4. Abdulla J, Asferg C and Kofoed KF. Prognostic value of absence or presence of coronary artery disease determined by 64-slice computed tomography coronary angiography a systematic review and meta-analysis. Int J Cardiovasc Imaging. 2011;27:413-20.

5. Aldous S, Elliott J, McClean D, Puri A and Richards AM. Outcomes in Patients Presenting with Symptoms Suggestive of Acute Coronary Syndrome with Elevated Cardiac Troponin but Non-obstructive Coronary Disease on Angiography. Heart Lung Circ. 2015;24:869-78.

6. Pasupathy S, Tavella R and Beltrame JF. The What, When, Who, Why, How and Where of Myocardial Infarction With Non-Obstructive Coronary Arteries (MINOCA). Circ J. 2016;80:11-6.

7. Pathik B, Raman B, Mohd Amin NH, Mahadavan D, Rajendran S, McGavigan AD, Grover S, Smith E, Mazhar J, Bridgman C, Ganesan AN and Selvanayagam JB. Troponin-positive chest pain with unobstructed coronary arteries: incremental diagnostic value of cardiovascular magnetic resonance imaging. Eur Heart J Cardiovasc Imaging. 2016;17:1146-52.

8. Brolin EB, Jernberg T, Brismar TB, Daniel M, Henareh L, Ripsweden J, Tornvall $P$ and Cederlund $\mathrm{K}$. Coronary plaque burden, as determined by cardiac computed tomography, in patients with myocardial infarction and angiographically normal coronary arteries compared to healthy volunteers: a prospective multicenter observational study. PLoS One. 2014;9:e99783.

9. Scalone G, Niccoli G and Crea F. Pathophysiology, diagnosis and management of MINOCA: an update. Eur Heart $\mathrm{J}$ Acute Cardiovasc Care. 2018:2048872618782414.

10. Pitts R, Daugherty SL, Tang F, Jones P, Ho PM, Tsai TT, Spertus J and Maddox TM. Optimal secondary prevention medication use in acute myocardial infarction patients with nonobstructive coronary artery disease is modified by management strategy: insights from the TRIUMPH Registry. Clin Cardiol. 2017;40:347-355.

1- Serviço de Cardiologia do HSI.

2- Cardiologista do Hospital Aliança da Bahia.

Endereço para correspondência:

gilsonfeitosafilho@yahoo.com.br 